\title{
ORIGINAL ARTICLE Infant frontal EEG asymmetry in relation with postnatal maternal depression and parenting behavior
}

\author{
DJ Wen ${ }^{1}$, NN Soe ${ }^{1}$, LW Sim ${ }^{2}$, S Sanmugam ${ }^{2}$, K Kwek $^{3}$, Y-S Chong ${ }^{2,4}$, PD Gluckman ${ }^{2}$, MJ Meaney ${ }^{2,5,6}$, A Rifkin-Graboi ${ }^{2}$ and A Qiu ${ }^{1,2}$
}

Right frontal electroencephalogram (EEG) asymmetry associates with negative affect and depressed mood, which, among children, are predicted by maternal depression and poor parenting. This study examined associations of maternal depression and maternal sensitivity with infant frontal EEG asymmetry based on 111 mother-6-month-infant dyads. There were no significant effects of postnatal maternal depression or maternal sensitivity, or their interaction, on infant EEG frontal asymmetry. However, in a subsample for which the infant spent at least 50\% of his/her day time hours with his/her mother, both lower maternal sensitivity and higher maternal depression predicted greater relative right frontal EEG asymmetry. Our study further showed that greater relative right frontal EEG asymmetry of 6-month-old infants predicted their greater negative emotionality at 12 months of age. Our study suggested that among infants with sufficient postnatal maternal exposure, both maternal sensitivity and mental health are important influences on early brain development.

Translational Psychiatry (2017) 7, e1057; doi:10.1038/tp.2017.28; published online 14 March 2017

\section{INTRODUCTION}

Maternal depression and poor parenting predict childhood behavioral and emotional difficulties. ${ }^{1-4}$ Compared to children of non-depressed mothers, children of depressed mothers display more developmental problems, ${ }^{5}$ greater stress system dysregulation, ${ }^{6}$ more negative affect, ${ }^{7}$ poorer affect regulation, ${ }^{8}$ more behavior problems, ${ }^{9,10}$ less cooperation ${ }^{11}$ and poorer social skills. ${ }^{9}$ Likewise, influences of poor maternal parenting behaviors are comparable to those of postnatal maternal depression and include negative emotionality, ${ }_{1}^{12}$ dysregulation of the stress responses, ${ }^{13,14}$ and poorer child behavior and socio-emotional function. ${ }^{15-22}$ Both maternal depression and parenting behaviors shape emotional and cognitive information, which affects child development in attention and memory as well as emotional reactivity of the central nervous system. ${ }^{23}$ Nevertheless, limited research has investigated whether both independently or interactively influence child brain development, especially for brain regions associated with emotion.

Associations of maternal depression and poor parenting with the aforementioned child outcomes are likely multifactorial. One possibility is that both maternal depression and poor parenting behaviors can influence children through separate behavioral approach and withdrawal systems. Depressive mothers who display a withdrawn style of interacting, express less positive affect and are more disengaged than non-depressed mothers. ${ }^{24}$ Depressed mothers who display an intrusive style, tend to overstimulate their infants by poking, restraining or aggressively introducing or withdrawing toys. ${ }^{25}$ These behaviors, in which depressed mothers fail to respond appropriately to infant emotional cues or to provide adequate levels of positive affect, may interfere with infant emotional development. ${ }^{26-28}$ After repeated failure to engage with their mothers, infants may withdraw from interactions with them. Infants may also use less mature regulatory strategies to cope with negative emotions, resulting in them experiencing more negative affect. ${ }^{29}$ Notably, these experiences in early life may lead to the engagement of the neural systems supporting withdrawal. ${ }^{30}$ On the other hand, one facet of parenting behaviors that can be assessed is maternal sensitivity, referring to the ability of a mother to perceive signals from her child and to interpret them accurately and respond promptly and appropriately. ${ }^{31}$ Maternal sensitivity is found to predict a variety of childhood socio-emotional constructs. ${ }^{15-22}$ This includes attachment security, a construct reflective of whether infants manage distress by approaching or avoiding their mothers. $^{32,33}$ Maternal sensitivity, may also be expected to influence offspring affective experience, as well as their approach-avoid patterns, which may shape the neural systems supporting approach.

Electroencephalogram (EEG) has been widely used to understand brain function in infants and children. EEG asymmetry reflects the difference between the EEG power observed at the right hemisphere and the left hemisphere. ${ }^{34}$ Convergent evidence shows that left frontal asymmetry of activation is associated with trait tendencies toward a general approach, or behavioral activation motivational system or positive emotion, while right frontal asymmetry of activation is associated with trait tendencies toward a general avoidance or withdrawal system, or negative emotion. ${ }^{35-37}$ Indeed, right frontal asymmetry of neural activity has been found to be linked with behavioral inhibition and social withdrawal in infants, children and adults. ${ }^{38,39}$ Likewise, infants of depressed mothers show greater relative right frontal asymmetry compared to infants of non-depressed mothers. ${ }^{40-43} \mathrm{~A}$ metaanalysis confirmed right frontal EEG asymmetry in children as a marker of the presence of familial stressors across a wide range of

\footnotetext{
${ }^{1}$ Department of Biomedical Engineering, Clinical Imaging Research Center, National University of Singapore, Singapore, Singapore; ${ }^{2}$ Singapore Institute for Clinical Sciences, Singapore, Singapore; ${ }^{3} \mathrm{KK}$ Women's and Children's Hospital, Singapore, Singapore; ${ }^{4}$ Department of Obstetrics \& Gynaecology, Yong Loo Lin School of Medicine, National University of Singapore, Singapore, Singapore; ${ }^{5}$ Ludmer Centre for Neuroinformatics and Mental Health, Douglas Mental Health University Institute, McGill University, Montréal, QC, Canada and ${ }^{6}$ Sackler Program for Epigenetics \& Psychobiology, McGill University, Montréal, QC, Canada. Correspondence: Professor A Qiu, Department of Biomedical Engineering, Clinical Imaging Research Center, National University of Singapore, 4 Engineering Drive 3, Block E4 \#04-08, Singapore 117583, Singapore.

E-mail: bieqa@nus.edu.sg

Received 6 December 2016; revised 10 January 2017; accepted 16 January 2017
} 
samples. ${ }^{44}$ Patterns of frontal EEG are proposed to serve as markers for the incidence of mood disorders in children ${ }^{45}$ and to predict behavioral and emotional problems. ${ }^{46}$ Moreover, reduced maternal sensitivity is associated with greater relative right frontal asymmetry. ${ }^{47}$

Although many studies show that depressed women display poor parenting, not all depressed mothers are necessarily ineffective in their parenting. For example, Zahn-Waxler et al. ${ }^{48}$ find that maternal parenting behavior moderated the association between maternal depression and child behavior problems. Depressed mothers who use proactive childrearing approaches have children who show fewer externalizing problems compared to those whose mothers use more negative reactive approaches. Given such findings, one question of interest is whether maternal sensitivity moderates the effects of maternal depression on infant frontal EEG asymmetry.

In addition, while to date, a large portion of literature focuses on maternal depression or maternal sensitivity and their relations with child outcomes, few studies investigate the time that mothers spend with their children. This oversight may be partly due to the longstanding notion that mothers serve as primary caregiver during infancy. ${ }^{49,50}$ Moreover, cross-species data suggest that infant expectations about the world are initially shaped by exposure to variations in maternal behavior. ${ }^{51}$ However, the degree to which mothers are present with infants may vary by generation, culture norms, , $^{52}$ socioeconomic conditions ${ }^{53,54}$ and maternal employment status. ${ }^{55,56}$ For example, time diaries of large samples show that the average time employed women spend caring for their children is less than non-employed women. ${ }^{55,56}$ Furthermore, in many Asian cultures, it is common for infants to live in the same household as their co-residential grandparents or to be extensively cared for by their grandparents. ${ }^{57,58}$ Key to the aforementioned argument is the possibility that exposure to frequent non-optimal care may solidify into trait-like differences in affect, motivation, and, so, frontal EEG asymmetry. A large portion of mothers in Southeast Asian countries are employed (in our sample, $73.1 \%$ of mothers are working). Hence, it is important to consider the amount of time infants spent with their mothers when investigating the influence of exposure to non-optimal caregiving on infant frontal function.

The present study aimed to examine the effects of maternal sensitivity and postnatal maternal depression on frontal EEG asymmetry of offspring at 6 months of age, while considering the amount of time the infants spend with their mothers. Substantial literature has suggested the relationship of maternal depression and right frontal EEG asymmetry of infants and relatively fewer studies investigate the association between maternal sensitivity and frontal EEG asymmetry of infants. However, no studies to date have explored the possible moderation of the relation between maternal depression and infant frontal EEG asymmetry by maternal sensitivity. We were interested in exploring whether exposure to postnatal depression and low maternal sensitivity were independently sufficient to predict right frontal EEG asymmetry, or whether variation in one of these maternal variables might enhance, or buffer against, the other. Hence, in this study, we used data from a longitudinal birth cohort based in Singapore to examine whether maternal depression and maternal sensitivity operate interactively or independently to influence frontal EEG asymmetry. In addition, this cohort study capitalizes on a 'natural experiment' occurring within the Singaporean caregiving context, allowing us to examine the degree to which time spent with biological mothers influences the aforementioned relations. We hypothesized that postnatal maternal depression and maternal sensitivity influence infant frontal EEG asymmetry only when the time infants spent with their mother is above a certain amount. Finally, given the fact on the association between right frontal EEG asymmetry and negative emotion, we examined whether such an association was also observed in our sample.

\section{MATERIALS AND METHODS}

\section{Participants}

Participants were recruited from Growing Up in Singapore Towards Healthy Outcomes (GUSTO), a longitudinal, Singaporean birth cohort study. ${ }^{59}$ The GUSTO cohort consisted of pregnant Asian women attending the first trimester prenatal ultrasound scan clinic at the National University Hospital (NUH) and KK Women's and Children's Hospital (KKH) in Singapore. The parents were Singapore citizens or Permanent Residents of Chinese, Malay or Indian ethnic background. The GUSTO cohort study was approved by the National Healthcare Group Domain Specific Review Board (NHG DSRB) and the Sing Health Centralized Institutional Review Board (CIRB). In addition, written consent was obtained from mothers.

Socioeconomic status (household income) was extracted from survey questionnaires conducted as part of a scheduled appointment during pregnancy. Birth outcome and pregnancy measures were obtained from hospital record. The present study included healthy term-born infants with gestational age $\geqslant 37$ weeks, birth weight $\geqslant 2.5 \mathrm{~kg}$, and a 5 -min neonatal Appearance, Pulse, Grimace, Activity and Respiration (APGAR) score $\geqslant 9$. These inclusion criteria were applied to limit the influence of variation in maturational effects on the fetal brain development. The full sample of the current study included 111 infants who fulfilled the above inclusion criteria and had good EEG, maternal sensitivity data and maternal depression questionnaire data.

\section{Maternal depression}

The Edinburgh Postnatal Depression Scale (EPDS) questionnaire was administered to mothers at 26 weeks of gestation and 3 months after delivery, and was used to quantify prenatal and early postnatal levels of maternal depressive symptomatology. The EPDS is a widely used 10-item self-report scale designed as a screening instrument for postnatal depression and has been well validated for use in prenatal and postnatal depression. ${ }^{60}$ Each item in the EPDS is scored on a four-point scale $(0-3)$ and items 3 and 5 to 10 are reverse scored. Higher scores indicate a greater intensity of depressive symptoms. The reliability of the EPDS scores assessed using Cronbach's analysis was 0.85 and 0.82 for the prenatal and postnatal EPDS for our cohort, respectively.

\section{Maternal sensitivity}

A 15-min mother-child interaction was recorded as part of a 3-h laboratory visit when infants were 6 months of age ( \pm 2 weeks). The mother was asked to 'interact or play' with her 6-month old infant 'as she normally would at home'. The one-way mirrored room was equipped with a foldable chair, highchair and a mat, but no toys for the first $5 \mathrm{~min}$. After $5 \mathrm{~min}$, a standard set of attractive toys and books was brought into the room. Maternal sensitivity was assessed using the Revised Mini-A short form of the Maternal Behavioral Q-Sort-V (Mini-MBQS-V). ${ }^{61,62}$ The Mini-MBQS-V consists of 25 items, each representing different possible aspects of sensitive and insensitive maternal behavior during interaction with an infant. The two Southeast Asian coders who scored the majority of the current study's cases were directly trained by the developers of the Mini-MBQS-V coding system (D. Pederson and S. Bento). The local coders were fluent in both English and the predominant mother tongue languages of Singapore, with one coder fluent in both English and Tamil, and the other in English, Malay and Mandarin. The two local coders achieved a high inter-rater correlation $(r=0.86)$ on roughly $15 \%(n=64)$ of coded cases from the larger GUSTO sample ( $n=424$ coded to date).

\section{Time infants spent with mothers}

A self-administered questionnaire capturing information on caregiver involvement and infants' sleeping pattern was given to mothers when infants were 6 months of age ( \pm 2 weeks). The mothers were asked to complete the questionnaire based on a 'typical week' during the past month, first listing all the possible caregivers in relation to the infant. We defined 'caregivers' as one who spends at least $2 \mathrm{~h}$ of time with the infant during the week and is responsible for aspects of the infant's daily routine such as feeding, bathing, playing, reading, changing diapers, cuddling and bedtime routines. Participants were then asked to provide detailed information on the infant's sleeping pattern during a 'typical week', and to also list the caregivers who were present during the infant's 'nonnocturnal sleeping hours', that is, hours during the day, inclusive of napping time. If more than one caregiver were listed, participants were then asked to note the one caregiver who is most likely to attend to the 
infant if he/she is upset or in need during his/her waking hours. We derived the percentage of time infants spent with their mothers during the day, by using the following rationale:

Percentage of time mothers spent with infant

$$
=100 \times \frac{\text { total hours in a typical week, mothers were listed present with infant }}{\text { total hours of infant's non }- \text { nocturnal sleeping duringa typical week }}
$$

The same rationale was then used to derive the time infants spent with the alternative caregivers (that is, father, maternal grandfather, maternal grandmother, paternal grandfather, paternal grandmother, domestic helper and other caregivers (for example, other relatives or infant care)) by replacing the time infants spent with mothers by the time the infants spent with the alternative caregiver.

\section{Infant toddler socio-emotional assessment}

Mothers reported emotional behavior of their 12-month-old infants using the 'Infant Toddler Socio-Emotional Assessment' (ITSEA) questionnaire. ${ }^{63}$ The ITSEA is a reliable and validated test for negative emotionality at this age. A higher score on the negative emotionality domain may indicate greater problems with emotion of infants.

\section{EEG preprocessing and spectral power analysis}

The EEG recording procedure was described elsewhere. ${ }^{64}$ Briefly, the 128 channel Geodesic Sensor Nets connected to a DC-coupled amplifier (Net Amp 300, Electrical Geodesic, Eugene, OR, USA) were used to measure EEG signals during the first $2 \mathrm{~min}$ of resting state and subsequent $38 \mathrm{~min}$ of a passive auditory oddball task in infants at 6 months of age. The first 2-min recording was for the purpose of stabilizing the EEG signal. As it is a common practice in infant research, four eye channels $(125,126,127,128)$ were removed to increase infant tolerance of the procedure. EEG was thus recorded from 124 channels out of 128 channels. At recording, all channels were referenced to vertex $\mathrm{Cz}$ position. The signal from all the channels was digitized at $250 \mathrm{~Hz}$ and filtered using a band-pass filter with the frequency range of $0.1-100 \mathrm{~Hz}$.

For EEG processing, we first removed 25 channels $(1,8,14,17,21,25,32$, $38,44,48,49,56,63,68,73,81,82,88,89,94,99,107,113,114,119)$, as they are located at the outer ring of the EEG net (Figure 1) and their signals were flat or with motion artifacts. For the rest of the EEG channels $(n=99)$, artifacts, including eye blinks and muscle movements, were identified via visual inspection and removed using the EEGLAB toolbox. ${ }^{65}$ Each channel's signal was re-referenced using an average referencing configuration. The mean voltage was subtracted from each channel to eliminate $D C$ effects. Artifacts associated with motor movement over $150 \mu \mathrm{V}$ peak to peak were also eliminated from all subsequent analyses. Finally, we selected the first 10-min segment of EEG signals after the 2-min resting EEG recording, as this was the longest good continuous EEG segment that can be extracted from all subjects.

The absolute power spectrum of each channel that ranged in the infant alpha frequency band $(6-9 \mathrm{~Hz})$ was computed, using a discrete Fourier transform with a Hamming window of a $2 \mathrm{~s}$ wide epoch and a $50 \%$ overlap between epochs. The power spectra were then log-transformed and averaged across the frontal left (FL) channels $(12,19,20,23,24,26,27,28$, $33,34)$ and frontal right (FR) channels $(2,3,4,5,116,117,118,122,123,124$; see Figure 1). A higher power spectrum value represents lower neural activity. ${ }^{66}$ Frontal asymmetry (FA) power scores were computed as follows, $\mathrm{FA}=(\mathrm{FR}-\mathrm{FL}) /(\mathrm{FR}+\mathrm{FL})$. This calculation method has been used in several other studies ${ }^{67-70}$ to quantify EEG power asymmetry. It has been shown that this asymmetry measure approximately follows Gaussian distribution for the following statistical testing. ${ }^{71,72}$ A positive FA value reflects greater relative left frontal neural activity than right frontal neural activity (relative left frontal asymmetry), while its negative value reflects greater relative right frontal neural activity than left frontal neural activity (relative right frontal asymmetry).

\section{Statistical analysis}

Multiple regression analyses were used to examine the independent and/ or interactive contributions of maternal depression and sensitivity on frontal EEG asymmetry. Postnatal maternal depression and maternal sensitivity as predictors were initially centered to minimize multicollinearity, and their interaction was formed as the product of the two centered predictors. A hierarchical order of entry was used to enter predictors. Covariates were entered in the first block. The second block included postnatal maternal depression, maternal sensitivity and their interaction. In cases where the interaction term was not significant, a reduced regression model was used to consider independent effects of postnatal maternal depression and maternal sensitivity on frontal EEG asymmetry.

To determine which covariates to enter into the models, we screened the relations between frontal EEG asymmetry and potentially relevant variables (for example, gender, gestational age, birth-weight, prenatal smoking exposure, prenatal alcohol exposure, maternal ethnicity, maternal age at delivery, household income, maternal education and infant age on the EEG visit day (number of days from birth to the visit day)). These variables were considered as potential covariates because of their possible contributions to infant frontal EEG asymmetry. For example, gender differences in frontal EEG asymmetry have been found in infants. ${ }^{73}$ Prenatal smoking exposure and prenatal alcohol exposure were more related to the mother's health. Studies have shown that smoking exposure influences frontal EEG asymmetry in adults. ${ }^{74}$ Maternal ethnicity, maternal age, household income and maternal education were related to socioeconomic status of the infant's family. Given that socioeconomic status was found to be related to adolescent frontal EEG asymmetry, ${ }^{75}$ we examined these variables as potential covariates. In our sample, maternal ethnicity and maternal age were significantly $(P<0.05)$ associated with frontal EEG asymmetry and were entered as covariates. In addition, as past research indicated the effects of prenatal maternal depression on frontal EEG asymmetry, ${ }^{76-78}$ prenatal maternal depression was also entered as a covariate. The whole process led to the inclusion of maternal ethnicity, maternal age and prenatal maternal depression as covariates in the regression analysis mentioned above. Covariates that had a categorical level of measurement (that is, maternal ethnicity) were dummy coded before they were entered into the regression model to ensure their suitability for regression.

Additional regression analysis was performed in the subsample selected based on the amount of time infants spent with their mothers. Since there is no clear cut-off for the mother time spent, we carried out a series of regressions to determine the minimum amount of mother time spent for the predictors (that is, maternal depression and maternal sensitivity) to have an effect on infant frontal EEG asymmetry. These regressions were carried out from at least 0 to $50 \%$ mother time spent. Regressions with mother time spent above $50 \%$ were not analyzed as the sample size becomes small for statistical power. The results of these series regression analyses (Figure 2) suggested that mothers needed to spend at least $50 \%$ of their infants' time for both maternal sensitivity and maternal depression to influence infant frontal EEG asymmetry. Hence, in the subsequent study, infants were split into two subsamples, those who spent at least $50 \%$ of their time with their mothers and those who did not.

Following this, regression analyses were performed for two groups of subjects: (1) the full sample and (2) the 'high mother time spent' subsample. The full sample referred to all the subjects that were included in the study regardless of the amount of time the infant had spent with the mother. The subsample only included infants who had spent at least $50 \%$ of their time during their own individualized daytime hours with their mothers.

\section{RESULTS}

Demographics

Out of 258 GUSTO infants who participated in the EEG recording: 64 infants did not have usable EEG data (for example, data with motion and muscle artifacts); 20 infants did not meet the inclusion criteria; 52 infants whose mothers did not complete questionnaire data (for example, time spent or EPDS questionnaire); and 11 infants whose mothers did not have maternal sensitivity data. Hence, the full sample of the current study included 111 infants. The correlations of maternal age, maternal ethnicity, maternal education, household income and maternal employment status with maternal depression were non-significant (all $P$-values $\geqslant 0.17$ ). Furthermore, among the aforementioned variables, only household income $(r=0.31, P=0.001)$ and maternal education $(r=0.25, P=0.008)$ were significantly correlated with maternal sensitivity.

Figure 3 shows the histogram of percentage of time infants spent with their mothers. The correlation between maternal employment status with mother time spent was significant 


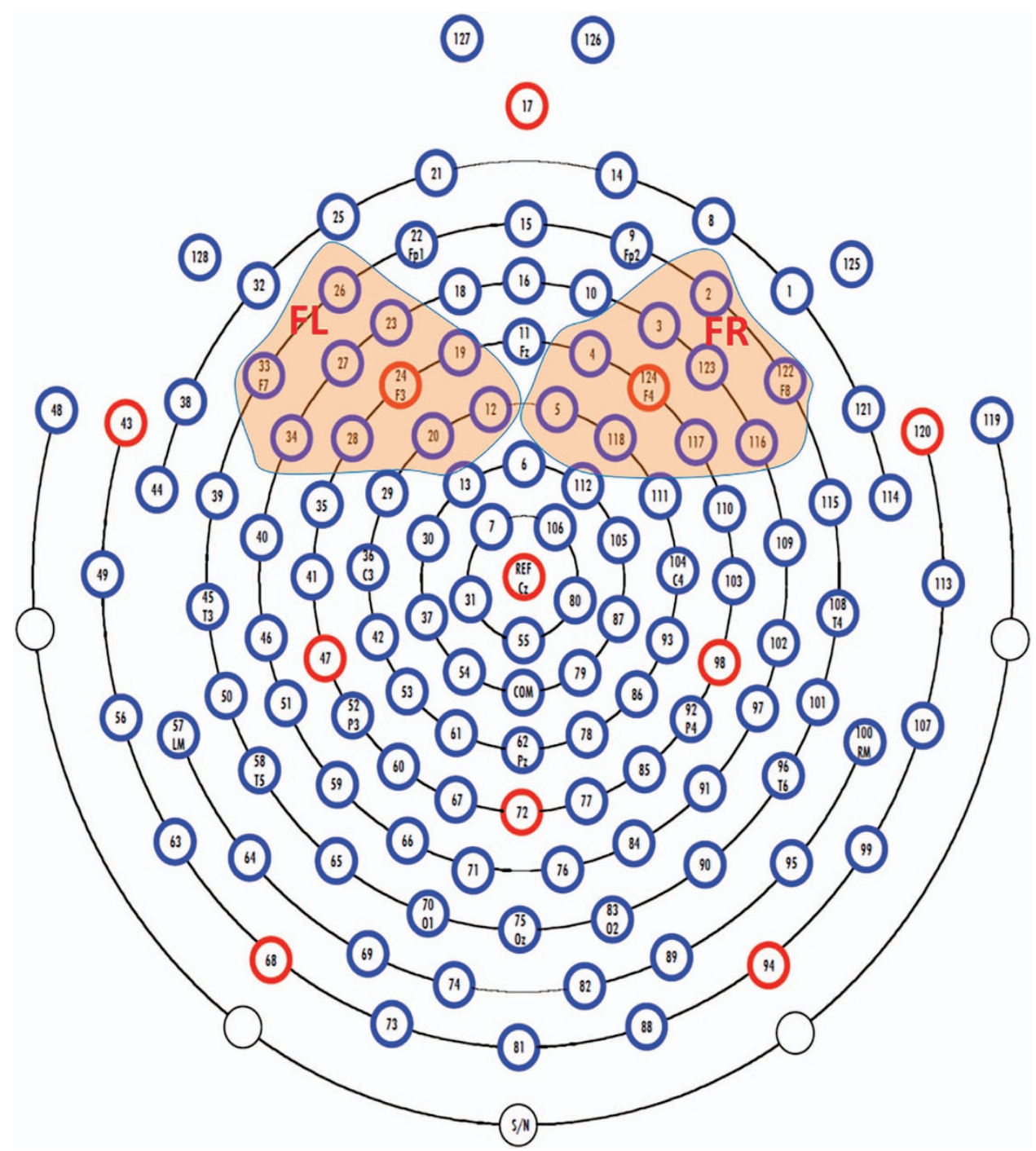

Figure 1. EGI HCGSN128 electrode net. The channels in the left and right frontal regions are highlighted in colored shading. FL, frontal left; FR, frontal right.

$(r=-0.625, P<0.001)$, while maternal age, maternal education and ethnicity were not related to mother time spent (all $P$-values $\geqslant 0.12$ ). Moreover, the correlations between maternal age, maternal education and ethnicity with employment status were not significant (all $P$-values $\geqslant 0.14$ ). However, the correlation between employment status and household income was significant $(r=0.207, P<0.036)$, suggesting that the mothers who were employed also possessed higher household income.

Among 111 infants, 56 mothers spent $<50 \%$ of their infants' time and the 'high mother time spent' subsample consisted of 55 infants. Table 1 lists the demographic information of the full sample $(0-100 \%$ time spent) as well as the 'high mother time spent' subsample (51-100\% time spent). For each caregiver, time spent with infants is also listed in Table 1. In the full sample and both subsamples, mothers had the highest percentage of time spent among all caregivers. The 'high mother time spent' subsample did not differ from the 'low mother time spent' subsample in terms of gestational age, birth weight, AGPAR score, post-conceptual age at EEG visit, frontal EEG asymmetry score, prenatal or postnatal maternal depression score, maternal sensitivity score or maternal age when assessed using independent samples $t$-tests (all $P$-values $\geqslant 0.11$ ). The 'high mother time spent' subsample also did not differ from the 'low mother time spent' subsample on categorical variables including gender and prenatal smoking exposure assessed using chi-square tests (all $P$ values $\geqslant 0.51)$, as well as maternal education, prenatal alcohol exposure and household income, assessed using Fisher's exact test (all $P$-values $\geqslant 0.35$ ). However, there was a significant difference in maternal ethnicity between the 'high mother time spent' group and 'low mother time spent' group as assessed using a Fisher's exact test $(P=0.012)$.

Among the plausible covariates mentioned in the Materials and Methods section, only maternal ethnicity $(r=0.223, P=0.018)$ and maternal age $(r=-0.190, P=0.046)$ were found to significantly correlate with frontal EEG asymmetry. Furthermore, these variables were not found to be correlated to each other. As such, maternal ethnicity and maternal age were added to the analysis as covariates.

Frontal EEG asymmetry in relation with maternal depression and sensitivity

The results of the regression analysis (Tables 2 and 3 ) on the full sample showed no significant interaction effect between maternal sensitivity and postnatal maternal depression on frontal EEG 

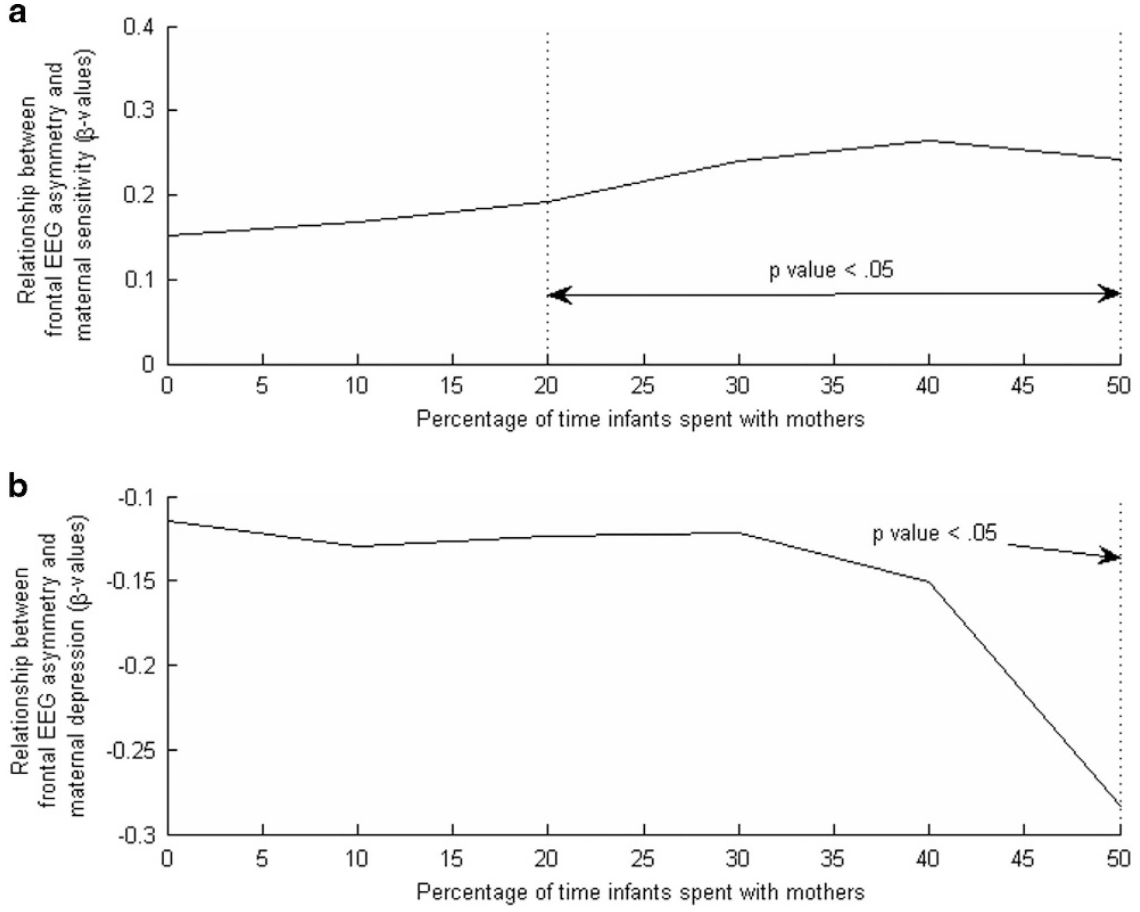

Figure 2. Associations of maternal sensitivity (a) and maternal depression (b) with infant frontal EEG asymmetry in the subsample with the percentage of time infants spent with their mothers. EEG, electroencephalogram.

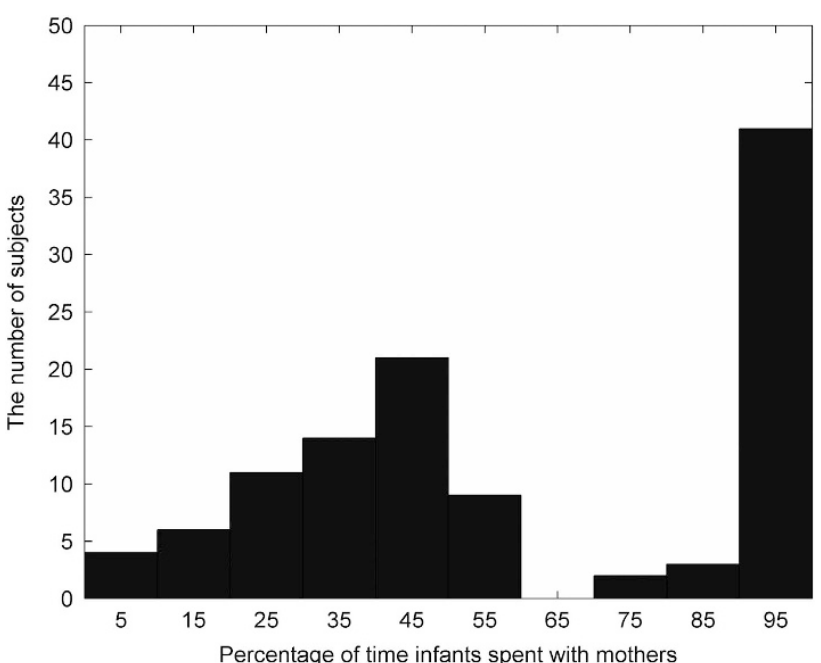

Figure 3. Histogram of the percentage of time infants spent with their mothers.

asymmetry $(\beta=0.061, \mathrm{df}=103, P=0.49)$. Likewise, there were no significant effects of maternal sensitivity $(\beta=0.152, d f=104$, $P=0.10)$ or postnatal maternal depression $(\beta=-0.114, \mathrm{df}=104$, $P=0.29)$ on infant frontal EEG asymmetry.

Within the 'high mother time spent' subsample, there was no significant interaction effect $(\beta=-0.082, \mathrm{df}=47, P=0.52)$. However, our study revealed that maternal sensitivity $(\beta=0.243$, $\mathrm{df}=48, P=0.04)$ and postnatal maternal depression $(\beta=-0.283$, $\mathrm{df}=48, P=0.04)$ were significantly associated with frontal EEG asymmetry. These findings (Tables 2 and 3 ) suggested that lower maternal sensitivity and higher postnatal maternal depression were associated with greater relative right frontal EEG asymmetry. Figure 4 shows the scatter plots of maternal sensitivity on frontal
EEG asymmetry and postnatal depression on frontal EEG asymmetry for the 'high mother time spent' subsample.

Frontal EEG asymmetry in relation with negative emotionality Pearson's correlation analysis revealed significant correlation between frontal EEG asymmetry and negative emotionality in the full sample $(r=-0.255, P=0.035, n=69)$, suggesting that greater relative right frontal EEG asymmetry of infants at 6 months of age was associated with greater negative emotionality of infants at 12 months of age. The similar trend was also found in the 'high mother time spent' subsample $(r=-0.283, P=0.116, n=32)$. This non-significant correlation in the subsample was mainly due to a relatively small sample size even though the correlation coefficient was greater than that from the full sample.

\section{DISCUSSION}

The present study revealed significant associations between both postnatal depression and maternal sensitivity on frontal EEG asymmetry that were apparent only in infants with 'high mother time spent'. No such effects were apparent in the full sample, where the amount of time that infants spent with their mothers ranged from 0 to $100 \%$. We found no evidence for an interaction effect between postnatal maternal depression and maternal sensitivity in infant EEG frontal asymmetry in both in either the full sample or the 'high mother time spent' subsample. These findings suggest that for infants who spend at least $50 \%$ of time with their mothers, postnatal maternal depression and maternal sensitivity exert similar, but independent effects on frontal EEG asymmetry. That is, among these infants, lower maternal sensitivity and greater maternal depression predicted greater relative right frontal EEG asymmetry. Such greater relative right frontal EEG asymmetry in 6-month-old infants also predicted their greater negative emotionality at 12 months of age.

In this study, maternal depression and insensitive parenting did not interactively predict right frontal EEG asymmetry in both the 
Table 1. Demographics of the full sample, 'high mother time spent' subsample and 'low mother time spent' subsample

\begin{tabular}{|c|c|c|c|}
\hline Measure & Full sample $(\mathrm{N}=111)$ & $\begin{array}{l}\text { 'High mother time spent' } \\
\text { subsample }(\mathrm{N}=55)\end{array}$ & $\begin{array}{c}\text { 'Low mother time spent' } \\
\text { subsample }(\mathrm{N}=56)\end{array}$ \\
\hline \multicolumn{4}{|l|}{ Infant characteristics } \\
\hline Gestational age (week), mean (s.d.) & $39.02(1.01)$ & $39.03(1.10)$ & $39.02(0.92)$ \\
\hline Birth weight $(\mathrm{kg})$, mean (s.d.) & $3.16(0.38)$ & $3.14(0.39)$ & $3.18(0.36)$ \\
\hline APGAR, mean & 9 & 9 & 9 \\
\hline Gender, male/female & $49 / 62$ & $26 / 29$ & $23 / 33$ \\
\hline Age at EEG visit (week), mean (s.d.) & $26.39(0.95)$ & $26.37(1.00)$ & $26.40(0.90)$ \\
\hline \multicolumn{4}{|l|}{ Infant frontal EEG } \\
\hline Left frontal EEG power, mean (s.d.) & $12.90(2.29)$ & $13.05(2.44)$ & $12.76(2.14)$ \\
\hline Range & 5.22 to 18.09 & 5.22 to 18.09 & 7.50 to 17.74 \\
\hline Right frontal EEG power, mean (s.d.) & $10.40(2.20)$ & $10.61(2.34)$ & $10.21(2.05)$ \\
\hline Range & 3.39 to 15.96 & 3.39 to 15.96 & 5.86 to 15.18 \\
\hline Frontal EEG asymmetry, mean (s.d.) & $-0.11(0.07)$ & $-0.11(0.08)$ & $-0.11(0.06)$ \\
\hline Range & -0.33 to 0.14 & -0.33 to 0.14 & -0.29 to 0.13 \\
\hline \multicolumn{4}{|l|}{ Mother characteristics } \\
\hline EPDS Prenatal Maternal Depression score, mean (s.d.) & $7.29(4.42)$ & $7.96(4.29)$ & $6.63(4.49)$ \\
\hline Range & 0.00 to 21.00 & 0.00 to 21.00 & 0.00 to 19.00 \\
\hline EPDS Postnatal Maternal Depression score, mean (s.d.) & $6.59(4.75)$ & $6.78(4.66)$ & $6.41(4.87)$ \\
\hline Range & 0.00 to 21.00 & 0.00 to 19.00 & 0.00 to 21.00 \\
\hline Maternal sensitivity, mean (s.d.) & $0.26(0.46)$ & $0.21(0.48)$ & $0.30(0.44)$ \\
\hline Range & -0.76 to 0.90 & -0.76 to 0.82 & -0.62 to 0.90 \\
\hline Mother time spent (\%), mean (s.d.) & $61.47(32.56)$ & $90.40(17.37)$ & 33.07 (12.89) \\
\hline Maternal age (year), mean (s.d.) & $30.27(4.71)$ & $30.89(4.88)$ & $29.66(4.50)$ \\
\hline \multicolumn{4}{|l|}{ Maternal ethnicity, \% } \\
\hline Chinese & 58.6 & 54.5 & 62.5 \\
\hline Malay & 28.8 & 23.6 & 33.9 \\
\hline Indian & 12.6 & 21.8 & 3.6 \\
\hline \multicolumn{4}{|l|}{ Maternal education, $\%$} \\
\hline Primary school & 2.7 & 1.9 & 3.6 \\
\hline Secondary school & 18.2 & 24.1 & 12.5 \\
\hline Pre-university, diploma or technical course & 41.8 & 35.2 & 48.2 \\
\hline University undergraduate level & 33.6 & 33.3 & 33.9 \\
\hline Above university undergraduate level & 3.6 & 5.6 & 1.8 \\
\hline Prenatal maternal smoking exposure, $\%$ yes & 39.6 & 37.0 & 42.3 \\
\hline Prenatal maternal alcohol exposure, $\%$ yes & 6.4 & 5.6 & 7.3 \\
\hline \multicolumn{4}{|l|}{ Maternal employment status, $\%$} \\
\hline Employed & 73.1 & 48.1 & 96.4 \\
\hline Unemployed & 26.9 & 51.9 & 3.6 \\
\hline \multicolumn{4}{|l|}{ Family characteristics } \\
\hline \multicolumn{4}{|l|}{ Household income (S\$), \% } \\
\hline$\leqslant 999$ & 2.9 & 2.0 & 3.6 \\
\hline 1000-1999 & 9.5 & 12.0 & 7.3 \\
\hline 2000-3999 & 30.5 & 32.0 & 29.1 \\
\hline $4000-5999$ & 26.7 & 24.0 & 29.1 \\
\hline$\geqslant 6000$ & 30.5 & 30.0 & 30.9 \\
\hline \multicolumn{4}{|l|}{ Caregiver } \\
\hline Mother time spent (\%), mean (s.d.) & $61.47(32.56)$ & $90.40(17.37)$ & 33.07 (12.89) \\
\hline Father time spent (\%), mean (s.d.) & $29.62(24.37)$ & $29.13(27.50)$ & $30.10(21.08)$ \\
\hline Maternal grandfather time spent (\%), mean (s.d.) & $5.87(20.82)$ & $3.28(15.23)$ & $8.41(25.02)$ \\
\hline Maternal grandmother time spent (\%), mean (s.d.) & $13.17(29.15)$ & $5.01(17.45)$ & $21.18(35.61)$ \\
\hline Paternal grandfather time spent (\%), mean (s.d.) & $8.70(23.18)$ & $8.07(22.37)$ & $9.33(24.13)$ \\
\hline Paternal grandmother time spent (\%), mean (s.d.) & $16.27(32.30)$ & 13.94 (31.34) & $18.57(33.34)$ \\
\hline Domestic helper time spent (\%), mean (s.d.) & $16.99(32.30)$ & $15.03(34.89)$ & $18.93(36.41)$ \\
\hline Other caregiver time spent (\%), mean (s.d.) & $14.59(27.81)$ & $8.56(20.86)$ & $20.50(32.37)$ \\
\hline
\end{tabular}

Abbreviations: APGAR, Appearance, Pulse, Grimace, Activity and Respiration; EEG, electroencephalogram; EPDS, Edinburgh Postnatal Depression Scale.

full sample and the 'high mother time spent' subsample. Our initial hypothesis was motivated by the finding that maternal parenting behaviors could moderate the effect of maternal depression on child behavior problems, ${ }^{48}$ where a large range of parenting behaviors including perspective taking, modulated control and promotion of prosocial approaches, were considered. Maternal sensitivity used in this study constituted only one facet of parenting and variation in sensitivity could be insufficient to moderate the effect of maternal depression on frontal EEG asymmetry. 
Table 2. Interaction effect of maternal sensitivity and postnatal maternal depression on infant frontal EEG asymmetry

\begin{tabular}{|c|c|c|c|c|}
\hline \multirow[t]{2}{*}{ Predictors } & \multicolumn{2}{|c|}{ Full sample } & \multicolumn{2}{|c|}{$\begin{array}{l}\text { 'High mother time } \\
\text { spent' subsample }\end{array}$} \\
\hline & $\Delta \mathrm{R}^{2}$ & $\beta$ & $\Delta \mathrm{R}^{2}$ & $\beta$ \\
\hline $\begin{array}{l}\text { Step } 1 \\
\quad \text { Covariates }\end{array}$ & $0.150^{* *}$ & & $0.265^{* *}$ & \\
\hline $\begin{array}{l}\text { Step } 2 \\
\text { Maternal sensitivity } \\
\text { Maternal depression } \\
\text { Interaction }\end{array}$ & 0.033 & $\begin{array}{l}0.155 \\
-0.115 \\
0.061\end{array}$ & $0.122^{*}$ & $\begin{array}{l}0.238^{*} \\
-0.301^{*} \\
-0.082\end{array}$ \\
\hline $\begin{array}{l}\text { Total } R^{2} \\
N\end{array}$ & $\begin{array}{c}0.182 \\
111\end{array}$ & & $\begin{array}{c}0.386 \\
55\end{array}$ & \\
\hline $\begin{array}{l}\text { Abbreviation: EEG, electro€ } \\
\text { level. Covariates: maternal } \\
\text { depression. }\end{array}$ & $\begin{array}{l}\text { ephalog } \\
\text { inicity, }\end{array}$ & $\begin{array}{l}\text { n. Note: } \\
\text { ternal as }\end{array}$ & $\begin{array}{l}<0.05 \text { le } \\
\text { and prer }\end{array}$ & $\begin{array}{l}{ }^{* * P} P<0.01 \\
\text { I maternal }\end{array}$ \\
\hline
\end{tabular}

Table 3. Independent effects of maternal sensitivity and postnatal maternal depression on infant frontal EEG asymmetry

\begin{tabular}{|c|c|c|c|c|}
\hline \multirow[t]{2}{*}{ Predictors } & \multicolumn{2}{|c|}{ Full sample } & \multicolumn{2}{|c|}{$\begin{array}{l}\text { 'High mother time } \\
\text { spent' subsample }\end{array}$} \\
\hline & $\Delta \mathrm{R}^{2}$ & $\beta$ & $\Delta \mathrm{R}^{2}$ & $\beta$ \\
\hline $\begin{array}{l}\text { Step } 1 \\
\quad \text { Covariates }\end{array}$ & $0.150^{* *}$ & & $0.265^{* *}$ & \\
\hline $\begin{array}{l}\text { Step } 2 \\
\quad \text { Maternal sensitivity } \\
\text { Maternal depression }\end{array}$ & 0.029 & $\begin{array}{c}0.152 \\
-0.114\end{array}$ & $0.116^{*}$ & $\begin{array}{c}0.243^{*} \\
-0.283^{*}\end{array}$ \\
\hline $\begin{array}{l}\text { Total } R^{2} \\
N\end{array}$ & $\begin{array}{c}0.179 \\
111 \\
\end{array}$ & & $\begin{array}{c}0.381 \\
55\end{array}$ & \\
\hline
\end{tabular}

Abbreviation: EEG, electroencephalogram. Note: ${ }^{*} P<0.05$ level, ${ }^{*} P<0.01$ level. Covariates: maternal ethnicity, maternal age and prenatal maternal depression.
Similarly, in the full sample, we did not find an independent association of maternal depression or maternal sensitivity with infant frontal EEG asymmetry. Previous studies commonly investigate samples in which mothers were clinically depressed. ${ }^{40,79,80}$ Although our sample was a community-based sample, this difference in the depressive scale could partly explain the absence of independent effects between maternal depression and sensitivity on infant frontal EEG asymmetry. An alternative explanation stems from the fact that we utilized an Asian cohort based in Singapore, whereas most other studies utilized Caucasian cohorts. ${ }^{40,43}$ Given cultural differences in caregiving practices, ${ }^{52}$ it is possible that most of these other studies occurred in a context where mothers were commonly present with their infants, whereas caregiving in our study was more diverse. Mothers in our study may be primary caregivers, however, they may not spend sufficient time with infants for their mood and/or sensitivity to influence their infants' frontal EEG asymmetry.

Notably, in the 'high mother time spent' subsample both maternal sensitivity and postnatal maternal depression were associated with infant frontal EEG asymmetry and the direction of effects is consistent with a recent meta-analysis examining frontal EEG asymmetry. ${ }^{44}$ For example, Hane and colleagues found lower maternal sensitivity at nine months of age was associated with greater relative right frontal EEG asymmetry during infancy ${ }^{47}$ and at age three. ${ }^{81}$ Similarly, Dawson et al. ${ }^{40}$ reported that higher postnatal maternal depression was associated with greater relative right frontal EEG asymmetry. It is also important to consider that frontal EEG as well as neuroimaging studies reflect the impact of maternal mood across the normal range in shaping neural structure and function. ${ }^{82-84}$ As expected, our sample suggested that greater relative right frontal EEG asymmetry at 6 months of age predicted greater negative emotionality at 12 months of age. This is largely consistent with the previous finding on the prediction of frontal EEG asymmetry to behavioral and emotional problems. ${ }^{46}$ Hence, the results of this study could highlight the importance of early interventions for improving the quality of care, even in low-risk groups. Infant brain development can be positively impacted from spending considerable time with mothers who are highly sensitive or have low levels of depressive symptoms. This could shift the infant's frontal EEG asymmetry leftwards, which promotes more healthy, positive emotions and approach behavior. ${ }^{35,37}$ This could then lower the risk for mood
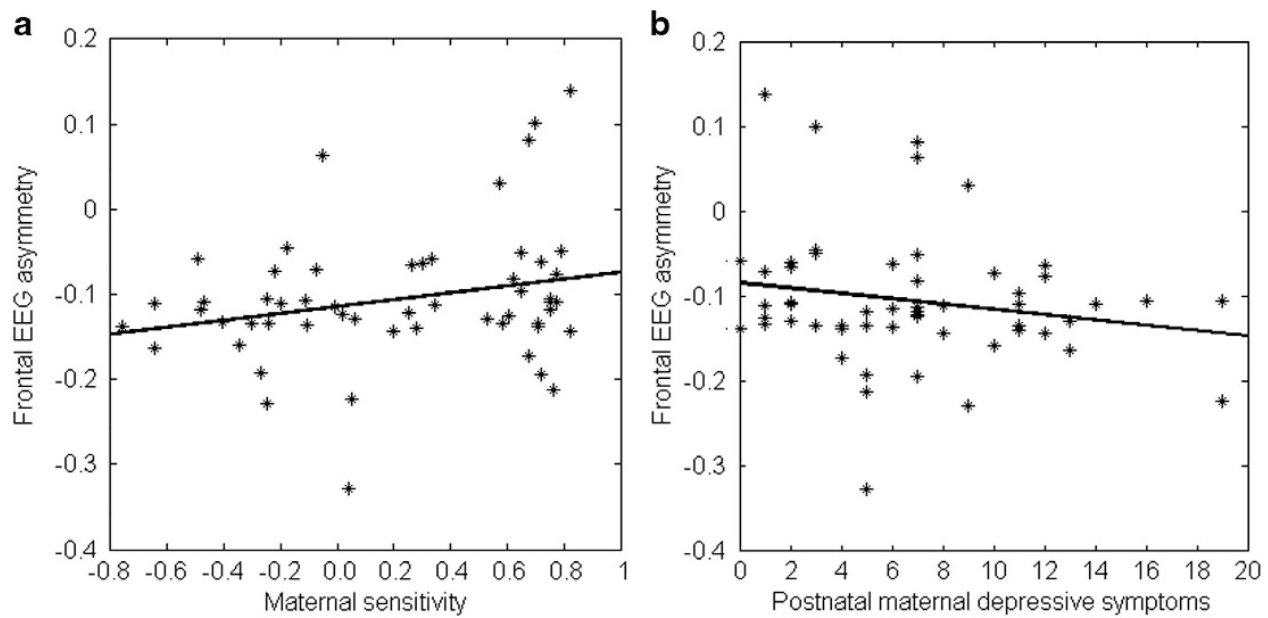

Figure 4. Relations of frontal EEG asymmetry with maternal sensitivity (a) and postnatal maternal depressive symptoms (b) in the 'high mother time spent' subsample. EEG, electroencephalogram. 
disorders ${ }^{45}$ and the likelihood of the infant exhibiting behavioral and emotional problems in childhood. ${ }^{46}$

\section{Limitations}

The study measured maternal sensitivity at 6 months, postnatal maternal depression at 3 months and frontal EEG asymmetry at 6 months. Furthermore, due to the consideration of subject burden, we only assessed prenatal depression at 26 weeks of gestation. Nevertheless, the second and third trimesters of pregnancy are critical periods when neural migration and synaptogenesis of the fetal brain occurs. In addition, our assessment of maternal depression was based on a common screening tool designed to elicit a subjective report of emotional well-being, but which did not constitute a clinical assessment. The reported results are thus best considered as being associated with self-reported depressive symptoms. We report a significant relation between both postnatal maternal depression and maternal sensitivity on infant's frontal function only when infants spent a substantial amount of time with their mothers. However, we cannot exclude the possibility that the limited relationship between these variables observed in the full sample was necessary due to decreased exposure to a depressed or insensitive mothers: such effects might reflect increased exposure to a nonmaternal 'nurturing' caregiver, and thus a protective effect. ${ }^{85}$ Finally, while our data suggest independent influences of postnatal maternal depression and maternal sensitivity, the nature of the effect of postnatal maternal depression remains to be defined and could involve forms of maternal care that are not reflected in the maternal sensitivity measure.

\section{CONCLUSION}

The present study focused on a community sample of mothers and infants and found that when infants spend a substantial amount of their time with their mothers, both maternal sensitivity and postnatal maternal depression symptomatology associate with infant frontal EEG asymmetry. These effects are consistent with the findings from previous studies such that the lower maternal sensitivity and higher postnatal maternal depression both predict greater relative right frontal EEG asymmetry. These results are interesting to consider in a cross-cultural context. Current practices in many countries substantially influence the amount of time mothers interact with their infants. ${ }^{52}$ In many Asian cultures, it is common for infants to live in the same household as their co-residential grandparents or to be extensively cared for by their grandparents. ${ }^{57,58}$ Thus, the association of maternal mood or maternal sensitivity on infant's frontal brain function may only be present when infants in Asian cultures spend a considerable amount of time with their mothers.

\section{CONFLICT OF INTEREST}

The authors declare no conflict of interest.

\section{ACKNOWLEDGMENTS}

The GUSTO study group includes Pratibha Agarwal, Arijit Biswas, Choon Looi Bong, Shirong Cai, Jerry Kok Yen Chan, Yiong Huak Chan, Cornelia Yin Ing Chee, Yin Bun Cheung, Audrey Chia, Amutha Chinnadurai, Chai Kiat Chng, Mary Foong-Fong Chong, Shang Chee Chong, Mei Chien Chua, Chun Ming Ding, Eric Andrew Finkelstein, Doris Fok, Keith M Godfrey, Anne Eng Neo Goh, Yam Thiam Daniel Goh, Joshua J Gooley, Wee Meng Han, Mark Hanson, Christiani Jeyakumar Henry, Joanna D Holbrook, ChinYing Hsu, Hazel Inskip, Jeevesh Kapur, Ivy Yee-Man Lau, Bee Wah Lee, Yung Seng Lee, Ngee Lek, Sok Bee Lim, Yen-Ling Low, Iliana Magiati, Lourdes Mary Daniel, Cheryl Ngo, Krishnamoorthy Naiduvaje, Wei Wei Pang, Boon Long Quah, Victor Samuel Rajadurai, Mary Rauff, Salome A Rebello, Jenny L Richmond, Lynette Pei-Chi Shek, Allan Sheppard, Borys Shuter, Leher Singh, Shu-E Soh, Walter Stunkel, Lin Lin Su, Kok Hian Tan, Oon Hoe Teoh, Mya Thway Tint, Hugo PS van Bever, Rob M van Dam, Inez
Bik Yun Wong, PC Wong, Fabian Yap, George Seow Heong Yeo. This study is supported by National Medical Research Council (NMRC; NMRC/TCR/004-NUS/2008, NMRC/TCR/012-NUHS/2014 and NMRC/CBRG/0039/2013), the Singapore Ministry of Education Academic Research Fund Tier 2 (MOE2012-T2-2-130) and by The JPB Foundation through The JPB Research Network on Toxic Stress, A Project of the Center on the Developing Child at Harvard University.

\section{REFERENCES}

1 Bohlin G, Hagekull B, Rydell AM. Attachment and social functioning: a longitudinal study from infancy to middle childhood. Soc Dev 2000; 9: 24-39.

2 Deater-Deckard K, Petrill SA. Parent-child dyadic mutuality and child behavior problems: an investigation of gene-environment processes. J Child Psychol Psychiatry 2004; 45: 1171-1179.

3 O'Connor TG, Heron J, Golding J, Glover V. ALSPAC Study Team Maternal antenatal anxiety and behavioural/emotional problems in children: a test of a programming hypothesis. J Child Psychol Psychiatry 2003; 44: 1025-1036.

4 Sroufe LA. The Development of the Person: The Minnesota Study of Risk and Adaptation from Birth to Adulthood. Guilford Press: New York, USA, 2005.

5 Deave T, Heron J, Evans J, Emond A. The impact of maternal depression in pregnancy on early child development. BJOG 2008; 115: 1043-1051.

6 Misri S, Grunau R, Weinberg J, Devlin AM, Papsdorf M, Oberlander TF. Prenatal exposure to maternal depression, neonatal methylation of human glucocorticoid receptor gene (NR3C1) and infant cortisol stress responses. Epigenetics 2008; 3: 97-106.

7 Society CP. Maternal depression and child development. Paediatr Child Health 2004; 9: 575-583.

8 DeMulder EK, Radke-Yarrow M. Attachment with affectively ill and well mothers: concurrent behavioral correlates. Development and psychopathology 1991; 3: 227-242.

9 Carter AS, Garrity-Rokous FE, Chazan-Cohen R, Little C, Briggs-Gowan MJ. Maternal depression and comorbidity: predicting early parenting, attachment security, and toddler social-emotional problems and competencies. J Am Acad Child Adolesc Psychiatry 2001; 40: 18.

10 Turney K. Pathways of disadvantage: explaining the relationship between maternal depression and children's problem behaviors. Soc Sci Res 2012; 41: 1546.

11 National Institute of Child Health and Human Development ECCRN. Chronicity of maternal depressive symptoms, maternal sensitivity, and child functioning at 36 months. Dev Psychol 1999; 35: 1297-1310.

12 Propper C, Moore GA. The influence of parenting on infant emotionality: a multilevel psychobiological perspective. Dev Rev 2006; 26: 427-460.

13 Atkinson L, Gonzalez A, Kashy DA, Santo Basile V, Masellis M, Pereira J et al. Maternal sensitivity and infant and mother adrenocortical function across challenges. Psychoneuroendocrinology 2013; 38: 2943-2951.

14 Bosquet Enlow M, King L, Schreier HM, Howard JM, Rosenfield D, Ritz T et al. Maternal sensitivity and infant autonomic and endocrine stress responses. Early Hum Dev 2014; 90: 377-385.

15 Bretherton I. The roots and growing points of attachment theory. In: Parkes CM, Stevenson-Hinde J, Marris P (eds). Attachment Across the Life Cycle. Tavistock/ Routledge: New York, NY, USA, 1991, pp 9-32.

16 Kerns KA. A developmental model of the relations between mother-child attachment and friendship. In: Gilmour RER (ed). Theoretical Frameworks for Personal Relationships. Lawrence Erlbaum Associates, Inc.: Hillsdale, NJ, USA, 1994, pp 129-156.

17 Raby KL, Roisman Gl, Fraley RC, Simpson JA. The enduring predictive significance of early maternal sensitivity: social and academic competence through age 32 years. Child Dev 2015; 86: 695-708.

18 Sroufe LA, Carlson E, Shulman S. Individuals in relationships: development from infancy through adolescence. In: Funder DC, Parke RD, Tomlinson-Keasey C, Widaman K (eds). Studying Lives Through Time: Personality and Development. American Psychological Association: Washington, DC, USA, 1993, pp 315-342.

19 Moilanen I, Ebeling H, Kumpulainen K, Raita-Hasu J, Kemppinen K. The continuity of maternal sensitivity from infancy to toddler age. J Reprod Infant Psychol 2006; 24: 199-212.

20 Stifter C, Spinrad T. Maternal sensitivity and infant emotional reactivity: concurrent and longitudinal relations. Marriage Fam Rev 2002; 34: 243-263.

21 Bernier A, Carlson SM, Whipple N. From external regulation to self-regulation: early parenting precursors of young children's executive functioning. Child Dev 2010; 81: 326-339.

22 Kok R, Lucassen N, Bakermans-Kranenburg MJ, van ljzendoorn $\mathrm{MH}$, Ghassabian A, Roza SJ et al. Parenting, corpus callosum, and executive function in preschool children. Child Neuropsychol 2014; 20: 583-606.

23 Gander M, Buchheim A. Attachment classification, psychophysiology and frontal EEG asymmetry across the lifespan: a review. Frontiers in Hum Neurosci 2015; 9: 79. 
24 Field T. Infants of depressed mothers. Dev Psychopathol 1992; 4: 49-66.

25 Jones NA, Field T, Fox NA, Davalos M, Malphurs J, Carraway $\mathrm{K}$ et al. Infants of intrusive and withdrawn mothers. Infant Behav Dev 1997; 20: 175-186.

26 Dawson G, Klinger LG, Panagiotides H, Spieker S, Frey K. Infants of mothers with depressive symptoms: Electroencephalographic and behavioral findings related to attachment status. Dev Psychopathol 1992; 4: 67-80.

27 Field T. Models for reactive and chronic depression in infancy. New Dir Child Dev 1986; 1986: 47-60.

28 Goodman SH, Gotlib IH. Risk for psychopathology in the children of depressed mothers: a developmental model for understanding mechanisms of transmission. Psychol Rev 1999; 106: 458-490.

29 Tronick EZ, Gianino JAF. The transmission of maternal disturbance to the infant. New Dir Child Dev 1986; 1986: 5-11.

30 Davidson RJ. Cerebral asymmetry, emotion, and affective style. In: Davidson RJ, Hugdahl K (eds). Brain Asymmetry. MIT Press: Cambridge, MA, USA, 1995.

31 Ainsworth MD. Patterns of Attachment: A Psychological Study of the Strange Situation. Lawrence Erlbaum Associates: New York; Hillsdale, NJ, 1978.

32 De Wolff MS, van ljzendoorn MH. Sensitivity and attachment: a meta-analysis on parental antecedents of infant attachment. Child Dev 1997; 68: 571-591.

33 Bowlby J. Attachment and Loss: Vol. 1. Attachment. Penguin: Harmondsworth, UK, 1969.

34 Field T, Diego M. Maternal depression effects on infant frontal EEG asymmetry. Int J Neurosci 2008; 118: 1081-1108.

35 Coan JA, Allen JJB. The state and trait nature of frontal EEG asymmetry in emotion. In: Hugdahl K, Davidson RJ (eds). The Asymmetrical Brain. MIT Press: Cambridge, MA, USA, 2003, pp 565-615.

36 Davidson R. Cerebral asymmetry and emotion: conceptual and methodological conundrums. Cogn Emot 1993; 7: 115-138.

37 Davidson RJ, Ekman P, Saron CD, Senulis JA, Friesen WV. Approach-withdrawal and cerebral asymmetry: Emotional expression and brain physiology I. J Pers Soc Psychol 1990; 58: 330.

38 Fox NA, Henderson HA, Rubin $\mathrm{KH}$, Calkins SD, Schmidt LA. Continuity and discontinuity of behavioral inhibition and exuberance: psychophysiological and behavioral influences across the first four years of life. Child Dev 2001; 72 : $1-21$.

39 Schmidt LA. Frontal brain electrical activity in shyness and sociability. Psychol Sci 1999; 10: 316-320.

40 Dawson G, Frey K, Panagiotides H, Osterling J, Hessl D. Infants of depressed mothers exhibit atypical frontal brain activity: a replication and extension of previous findings. J Child Psychol Psychiatry 1997; 38: 179-186.

41 Diego MA, Field T, Jones NA, Hernandez-Reif M. Withdrawn and intrusive maternal interaction style and infant frontal EEG asymmetry shifts in infants of depressed and non-depressed mothers. Infant Behav Dev 2006; 29: 220-229.

42 Diego MA, Jones NA, Field T. EEG in 1-week, 1-month and 3-month-old infants of depressed and non-depressed mothers. Biol Psychol 2010; 83: 7-14.

43 Jones NA, Field T, Almeida A. Right frontal EEG asymmetry and behavioral inhibition in infants of depressed mothers. Infant Behav Dev 2009; 32: 298-304.

44 Peltola MJ, Bakermans-Kranenburg MJ, Alink LRA, Huffmeijer R, Biro S, van ljzendoorn $\mathrm{MH}$. Resting frontal EEG asymmetry in children: meta-analyses of the effects of psychosocial risk factors and associations with internalizing and externalizing behavior. Dev Psychobiol 2014; 56: 1377-1389.

45 Coan JA, Allen JJB. Frontal EEG Asymmetry as a Moderator and Mediator of Emotion, vol. 67. Elsevier B.V: The Netherlands, 2004, pp 7-50.

46 Smith $C L$, Bell MA. Stability in infant frontal asymmetry as a predictor of toddlerhood internalizing and externalizing behaviors. Dev Psychobiol 2010; 52: 158-167.

47 Hane AA, Fox NA. Ordinary variations in maternal caregiving influence human infants' stress reactivity. Psychol Sci 2006; 17: 550-556.

48 Zahn-Waxler C, lannotti RJ, Cummings EM, Denham S. Antecedents of problem behaviors in children of depressed mothers. Dev Psychopathol 1990; 2: 271-291.

49 Bowlby J. Attachment and Loss: Vol. 1. Attachment. Hogarth Press and Institute of Psychoanalysis: London, 1969.

50 Bowlby J. Attachment and Loss: Vol. 2. Separation, Anxiety and Anger. Hogarth Press and the Institute for Psychoanalysis: London, 1969.

51 Cameron NM, Champagne FA, Parent C, Fish EW, Ozaki-Kuroda K, Meaney MJ. The programming of individual differences in defensive responses and reproductive strategies in the rat through variations in maternal care. Neurosci Biobehav Rev 2005; 29: 843-865.

52 Howes C, Spieker S. Attachment relationships in the context of multiple caregivers. In: Cassidy J, Shaver PR (eds). Handbook of Attachment: Theory, Research, And Clinical Applications, vol. 2. Guilford Press: New York, USA, 2008.

53 Kalil A, Ryan R, Corey M. Diverging destinies: maternal education and the developmental gradient in time with children. Demography 2012; 49: 1361-1383.
54 Sayer Liana C, Bianchi Suzanne M, Robinson John P. Are parents investing less in children? Trends in mothers' and fathers' time with children. Am J Sociol 2004 110: 1-43.

55 Bryant WK, Zick CD. Are we investing less in the next generation? Historical trends in time spent caring for children. J Fam Econ Issues 1996; 17: 365-392.

56 Gershuny J, Robinson JP. Historical changes in the household division of labor Demography 1988; 25: 537-552.

57 Chen F, Liu G, Mair CA. Intergenerational ties in context: grandparents caring for grandchildren in China. Soc Forces 2011; 90: 571-594.

58 Mehta KK, Thang LL. Experiencing Grandparenthood: An Asian Perspective, vol. 47 Springer Science+Business Media B.V: New York, USA, 2012 xiv-xivpp.

59 Soh S-E, Tint MT, Gluckman PD, Godfrey KM, Rifkin-Graboi A, Chan YH et al. Cohort profile: Growing Up in Singapore Towards healthy Outcomes (GUSTO) birth cohort study. Int J Epidemiol 2014; 43: 1401-1409.

60 Cox JL, Holden JM, Sagovsky R. Detection of postnatal depression. Development of the 10-item Edinburgh Postnatal Depression Scale. Br J Psychiatry 1987; 150: 782-786.

61 Moran G. Mini-MBQS-V revised mini-MBQS 25 item for video coding (2009). Available at: http://works.bepress.com/gregmoran/49/.

62 Tarabulsy GM, Provost MA, Bordeleau S, Trudel-Fitzgerald C, Moran G, Pederson DR et al. Validation of a short version of the maternal behavior Q-set applied to a brief video record of mother-infant interaction. Infant Behav Dev 2009; 32: 132-136.

63 Carter AS, Briggs-Gowan MJ. ITSEA: Infant-Toddler Social and Emotional Assessment. Pearson: San Antonio, TX, USA, 2006.

64 Cai S, Pang WW, Low YL, Sim LW, Sam SC, Bruntraeger MB et al. Infant feeding effects on early neurocognitive development in Asian children. Am J Clin Nutr 2015; 101: 326-336.

65 Delorme A, Makeig S. EEGLAB: an open source toolbox for analysis of single-trial EEG dynamics including independent component analysis. J Neurosci Methods 2004; 134: 9-21.

66 Lindsley DB, Wicke JD. The electroencephalogram: autonomous electrical activity in man and animals. In: Thompson R, Patterson M (eds). Bioelectric Recording Techniques. Academic Press: New York, 1974, pp 3-79.

67 Yuvaraj R, Murugappan M, Mohamed Ibrahim N, lqbal M, Sundaraj K, Mohamad K et al. On the analysis of EEG power, frequency and asymmetry in Parkinson's disease during emotion processing. Behav Brain Funct 2014; 10: 12-12.

68 Knott V, Mahoney C, Kennedy S, Evans K. EEG power, frequency, asymmetry and coherence in male depression. Psychiatry Res 2001; 106: 123-140.

69 Quraan MA, Protzner AB, Daskalakis ZJ, Giacobbe P, Tang CW, Kennedy SH et al. EEG power asymmetry and functional connectivity as a marker of treatment effectiveness in DBS surgery for depression. Neuropsychopharmacology 2014; 39 1270-1281.

70 Jansen A, Menke R, Sommer J, Förster AF, Bruchmann S, Hempleman J et al. The assessment of hemispheric lateralization in functional MRI-robustness and reproducibility. Neuroimage 2006; 33: 204-217.

71 Gasser T, Jennen-Steinmetz C, Sroka L, Verleger R, Möcks J. Development of the EEG of school-age children and adolescents II. Topography. Electroencephalogr Clin Neurophysiol 1988; 69: 100-109.

72 Martinović Z, Jovanović V, Ristanović D. EEG power spectra of normal preadolescent twins. Gender differences of quantitative EEG maturation. Neurophysiol Clin 1998; 28: 231-248.

73 Henderson HA, Fox NA, Rubin KH. Temperamental contributions to social behavior: the moderating roles of frontal EEG asymmetry and gender. J Am Acad Child Adolesc Psychiatry 2001; 40: 68-74.

74 Knott VJ, Naccache L, Cyr E, Fisher DJ, Mclntosh JF, Millar AM et al. Craving induced EEG reactivity in smokers: effects of mood induction, nicotine dependence and gender. Neuropsychobiology 2009; 58: 187-199.

75 Tomarken AJ, Dichter GS, Garber J, Simien C. Resting frontal brain activity: linkages to maternal depression and socio-economic status among adolescents. Biol Psychol 2004; 67: 77-102.

76 Field T, Diego M, Hernandez-Reif M, Schanberg S, Kuhn C. Relative right versus left frontal EEG in neonates. Dev Psychobiol 2002; 41: 147-155.

77 Field T. Prenatal Depression Effects on the Fetus and Neonate. Oxford University Press: Oxford, UK, 2004

78 Field T, Diego M, Dieter J, Hernandez-Reif M, Schanberg S, Kuhn C et al. Prenatal depression effects on the fetus and the newborn. Infant Behav Dev 2004; 27: 216-229.

79 Feng X, Forbes EE, Kovacs M, George CJ, Lopez-Duran NL, Fox NA et al. Children's depressive symptoms in relation to EEG frontal asymmetry and maternal depression. J Abnorm Child Psychol 2012; 40: 265.

80 Forbes EE, Shaw DS, Fox NA, Cohn JF, Silk JS, Kovacs M. Maternal depression, child frontal asymmetry, and child affective behavior as factors in child behavior problems. J Child Psychol Psychiatry 2006; 47: 79-87.

81 Hane AA, Henderson HA, Reeb-Sutherland BC, Fox NA. Ordinary variations in human maternal caregiving in infancy and biobehavioral development in early childhood: a follow-up study. Dev Psychobiol 2010; 52: 558. 
82 Qiu A, Anh T, Li Y, Chen H, Rifkin-Graboi A, Broekman BFP et al. Prenatal maternal depression alters amygdala functional connectivity in 6-month-old infants. Trans/ Psychiatry 2015; 5: e508.

83 Rifkin-Graboi A, Bai J, Chen H, WBr Hameed, Sim LW, Tint MT et al. Prenatal maternal depression associates with microstructure of right amygdala in neonates at birth. Biol Psychiatry 2013; 74: 837.

84 Soe NN, Wen DJ, Poh JS, Li Y, Birit FPB, Chen H et al. Pre- and post-natal maternal depressive symptoms in relation with infant frontal function, connectivity, and behaviors. PLOS ONE 2016; 11: e0152991.

85 Werner EE. Resilience in development. Curr Dir Psychol Sci 1995; 4: 81-85.
This work is licensed under a Creative Commons Attribution 4.0 International License. The images or other third party material in this article are included in the article's Creative Commons license, unless indicated otherwise in the credit line; if the material is not included under the Creative Commons license, users will need to obtain permission from the license holder to reproduce the material. To view a copy of this license, visit http://creativecommons.org/licenses/ by/4.0/

(c) The Author(s) 2017 